\title{
Reliability Modeling of Nanoelectronic Circuits
}

\author{
Jie Han, Erin Taylor, Jianbo Gao and José Fortes \\ Department of Electrical and Computer Engineering, University of Florida \\ Gainesville, Florida 32611-6200, USA. Email: \{jie, fortes $\} @$ ufl.edu
}

\begin{abstract}
Reliability and its modeling have become critical issues for nanotechnology-based circuits. This paper considers the use of probabilistic models of unreliable logic gates to estimate the reliability of nanoelectronic circuits and derive fundamental error bounds for logic gates. Two methods are experimentally contrasted with respect to accuracy and computational complexity.
\end{abstract}

Index Terms - Nanotechnology, nanoelectronics, reliability, probabilistic, fault-tolerance, simulation.

\section{INTRODUCTION}

As CMOS technology is predicted to reach its scaling limit in the next decade, various nanometer-scale devices have been investigated for future nanoelectronics beyond CMOS. Desirable characteristics of these devices may include extremely small dimensions, high switching speed, low power consumption or good scaling potential. However, shrinking device dimensions, smaller operating voltages/currents and the introduction of new materials have negative impacts on reliability and result in increased device failure rates. Physical failures of devices will inevitably cause malfunctions of logic gates and interconnects in a nanoelectronic circuit. Thus, reliability issues become important for nanoelectronic circuits composed of unreliable nano-devices.

A variety of fault-tolerant techniques have been investigated for nanoelectronic circuits and systems [1] [2]. Analytical approaches using Markov chains [3], Markov random fields (MRF) [4], bifurcation analysis [5] and probabilistic transfer matrices (PTMs) [6] have been proposed for the evaluation of circuit reliability. In this paper, we review our recent work on probabilistic methods for obtaining error bounds and reliability estimates of nanoelectronic circuits. A full adder is used as a modeling example due to its implementability using several promising nano-devices. Reliability estimates are compared to accurate results obtained by the PTM method. The advantages of each method are discussed.

\section{NANOELECTRONIC LOGIC AND CIRCUITS}

Having the merits of low power consumption and good scalability, single-electron tunneling (SET) technology has been extensively investigated and many SET-based circuits have been experimentally demonstrated. Fig. 1 shows a double-junction single-electron box (or a singleelectron trap) circuit [7]. This circuit consists of two socalled islands (or quantum dots), two tunnel junctions $C_{j}$ and capacitors. It functions as a complementary majority gate which produces an inverted majority output. However, a severe problem for SET circuits is that of random background noise. Impurities and trapped electrons in the substrate induce image charges $Q$ on the surface of the islands. A $Q$ comparable to a single electron charge will upset the correct state of a SET device.

Quantum-dot cellular automata (QCA) are based on the electrostatic interaction of QCA cells [8]. In a QCA cell single electrons reside in quantum dots and are able to tunnel through the barriers between dots. A majority gate based on four-dot QCA cells is shown in Fig. 2. In each cell two electrons tend to align along the diagonals due to their mutual electrostatic repulsion. As a result of the electrostatic interaction, the output cell of the QCA majority circuit takes the same polarization as a majority of those represented in the input cells. Errors in QCA circuits are mainly caused by displacements of cells [9]. QCA circuits also suffer from random background charges, since they use single-electron operations.

The physical failures of devices lead to a diversity of error behaviors in logic gates and interconnections. Conventional fault models at the gate level include stuckat faults, function inversion, bridging faults, transition faults, etc. Interconnect faults are usually shorts or opens. Shorts in interconnects are actually bridge faults that create a short between two or more lines, and opens in interconnects are assumed to behave as "soft" stuck-at-1's or stuck-at-0's. Another widely-used fault model, the von

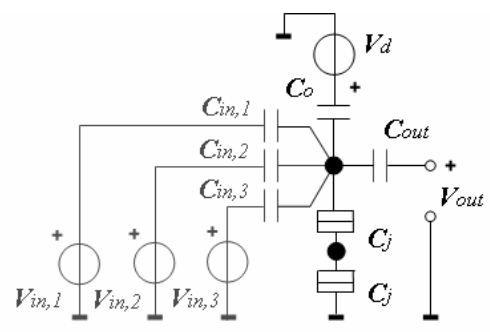

Fig. 1. A complementary majority gate in SET circuits. 

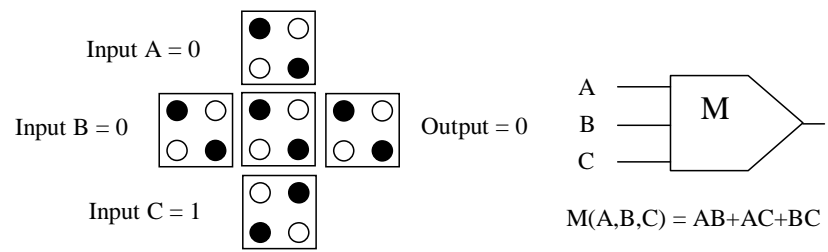

$\mathrm{M}(\mathrm{A}, \mathrm{B}, \mathrm{C})=\mathrm{AB}+\mathrm{AC}+\mathrm{BC}$

Fig. 2. A QCA majority logic and its schematic.

Neumann model, assumes that a gate flips its correct output. Since von Neumann faults generally result in the worst scenario for a circuit's reliability, this paper considers the von Neumann fault model.

In Fig. 3 a 1-bit full adder using five majority gates and three inverters is shown. This circuit has been proposed for an implementation using QCA cells [8]. Other fulladder designs have been proposed for implementations in SETs [10] and QCA [11], but the structure in Fig. 3 is used as our reliability modeling example.

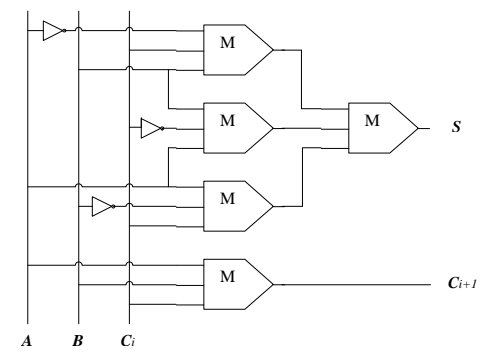

Fig. 3. A 1-bit full adder using majority gates and

\section{Probabilistic Models FOR LOGIC GATES}

In this section we present reliability models for unreliable logic gates. We employ two simple rules from the notion of Boolean ring, as used in the MRF method [4]. These rules relate the Boolean logic variables and the algebraic operations of their probabilities:

$$
X^{\prime} \rightarrow 1-X \quad \text { and } \quad X_{1} \wedge X_{2} \rightarrow X_{1} X_{2} .
$$

Let $X$ 's and $Z$ be the gate inputs and output, respectively. This method can be summarized as:

1. Construct a truth table for the logic gate;

2. Obtain a sum of the minterms for inputs that produce a " 1 " (or " 0 ") and its complement;

3. Consider the effect of a gate failure, and sum over the conditional probabilities with and without gate errors.

Let us take a majority gate as an example. Its truth values are in Table 1. According to the Boolean ring rules, the sum of the minterms for output " 1 " and " 0 " are

$Z^{\prime}=X_{1} X_{2}\left(1-X_{3}\right)+X_{1} X_{3}\left(1-X_{2}\right)+X_{2} X_{3}\left(1-X_{1}\right)+X_{1} X_{2} X_{3}$ and $1-Z^{\prime}$ respectively.
If each gate has a probability $\varepsilon$ of making a von Neumann error, the probability of its output being " 1 " is then given by

$$
\begin{aligned}
& Z_{V}=Z^{\prime}(1-\varepsilon)+\left(1-Z^{\prime}\right) \varepsilon= \\
& (1-\varepsilon)\left(X_{1} X_{2}+X_{1} X_{3}+X_{2} X_{3}-2 X_{1} X_{2} X_{3}\right)+ \\
& \varepsilon\left(1-2 X_{1} X_{2}-2 X_{1} X_{3}-2 X_{2} X_{3}+4 X_{1} X_{2} X_{3}\right)
\end{aligned}
$$

This method to construct a gate reliability model can be used for any type of gate and fault models. Further, an interconnect can be modeled as an identity gate, i.e. it reproduces its input as output. Hence, the reliability of an unreliable interconnect for a von Neumann fault can be obtained by following the above procedure as

$$
Z_{V}=X+\varepsilon(1-2 X)
$$

Note that in a nanoelectronic circuit, it may not be true that all gates and interconnects have the same error rate the fault mechanism and the rates of fault occurrence vary greatly depending on the actual implementation.

These gate models have been used to construct nonlinear mapping functions, from which fundamental error Table 1. Majority gate bounds can be derived [5]. Let us consider a network of NAND gates, and label a sequence of the gates by index $i, i=1,2, \cdots, \mathrm{n}$, $\cdots$, where the output of gate $i$ becomes an input to gate $i+1$. If the two inputs of any gate have equal probability of being " 1 ", the gate equation for NAND obtained from the above procedure reduces to a simple nonlinear map,

$$
X_{n+1}=(1-\varepsilon)+(2 \varepsilon-1) X_{n}{ }^{2}
$$
truth table.

\begin{tabular}{|c|c|c|c|}
\hline$X_{1}$ & $X_{2}$ & $X_{3}$ & $Z$ \\
\hline 0 & 0 & 0 & 0 \\
\hline 0 & 0 & 1 & 0 \\
\hline 0 & 1 & 0 & 0 \\
\hline 0 & 1 & 1 & 1 \\
\hline 1 & 0 & 0 & 0 \\
\hline 1 & 0 & 1 & 1 \\
\hline 1 & 1 & 0 & 1 \\
\hline 1 & 1 & 1 & 1 \\
\hline
\end{tabular}

In such a map, $\varepsilon$ is called a bifurcation (or controlling) parameter. The dynamic behavior of the map can be examined by so-called bifurcation analysis. Such a bifurcation analysis of (4) reveals that a period-doubling bifurcation occurs at:

$$
\varepsilon^{*}=0.08856
$$

indicating an error bound of 0.08856 for NAND gates. Bifurcation analysis for finding error threshold values can be extended to arbitrary logic gates [5].

\section{RELIABILITY MODELS}

Consider the $i$ th gate in a circuit. The gate's probabilistic gate model (PGM) obtained in Section III can be formulated as: 


$$
X_{i}=\left[\begin{array}{ll}
p_{i} & 1-p_{i}
\end{array}\right] \cdot\left[\begin{array}{c}
1-\varepsilon \\
\varepsilon
\end{array}\right],
$$

where $p_{i}$ is the sum of the minterms of inputs that produce a "1", and, for example, $p_{i}=X_{i-1}+X_{i-2}-2 X_{i-1} X_{i-2}$ for an XOR gate with inputs $X_{i-1}$ and $X_{i-2}$. Here $X_{i}$ is the output of gate $i$ and thus an input to another gate in the circuit. Since (4) can be applied to any logic gate, an iterative execution of this procedure from a circuit's inputs to outputs will produce a probability/reliability of an output.

In this modeling method, assuming that gate input/output signals are statistically independent, the "overall" reliability of a circuit can be obtained by multiplying the individual reliabilities of each circuit output. In the general case, the proposed approach using PGMs will thus lead to approximate results, since circuits with fanouts do not meet the assumption of statistical independence among signals.

To consider the correlations among signals, it is possible to model a circuit with the joint and conditional probabilities among signals. The success of this method depends on finding the proper transitional probabilities and the methodologies for efficiently computing the joint probabilities.

Concurrently with, and independently from, this work, a similar approach using the so-called probabilistic transfer matrices (PTMs) was proposed for the evaluation of circuit reliability [6]. The PTM method uses a matrix representation of gate errors and an exhaustive listing of inputs/outputs. For a NAND gate, for example, its PTM is given by

$$
P T M_{\text {nand }}=\left[\begin{array}{cc}
\mathcal{E} & 1-\varepsilon \\
\mathcal{E} & 1-\varepsilon \\
\varepsilon & 1-\varepsilon \\
1-\varepsilon & \varepsilon
\end{array}\right],
$$

and its output probability can be obtained by

$$
\left[\begin{array}{ll}
p_{0} & p_{1}
\end{array}\right]=\left[\begin{array}{llll}
p_{00} & p_{01} & p_{10} & p_{11}
\end{array}\right] P T M_{\text {nand }},
$$

where $p_{0}$ and $p_{1}$ are output probabilities for " 0 " and " 1 "; $p_{00}, p_{01}, p_{10}$ and $p_{11}$ are initial probabilities for inputs (00, $01,10,11)$. The gate PTMs are combined to form a circuit PTM. For gates connected in series, a combined PTM is obtained as the matrix product of the gate PTMs, while for gates connected in parallel, the tensor product of gate PTMs is used as the circuit PTM to present the joint probabilities of signals. A specific PTM is constructed for a fanout due to its special behavior.

Since the PTM evaluation is based on the exhaustive listing of input and output probabilities, a circuit with $m$ inputs and $n$ outputs requires a PTM with $2^{m+n}$ entries. The PTM representation can be compressed through the use of algebraic decision diagrams, but only a low compression rate is possible for a large circuit PTM with gate errors. We shall illustrate in the following that the evaluation method using PGMs can approximate the PTM method while requiring much less space and time.

\section{Simulation Results}

We have used both the PGM and PTM methods to study the reliability of the majority-based full adder, as shown in Fig. 4. The PGM results are the products of the reliabilities of Sum and Carry. Because of the symmetries inherent to the inputs, gates and the circuit itself, the full adder in Fig. 3 exhibits two different characteristics in terms of reliability. Per Fig. 3, the reliabilities obtained by the two methods are nearly identical. It is also shown that the reliability of the circuit starts to drop rapidly at a gate error rate of approximately $10^{-3}$.

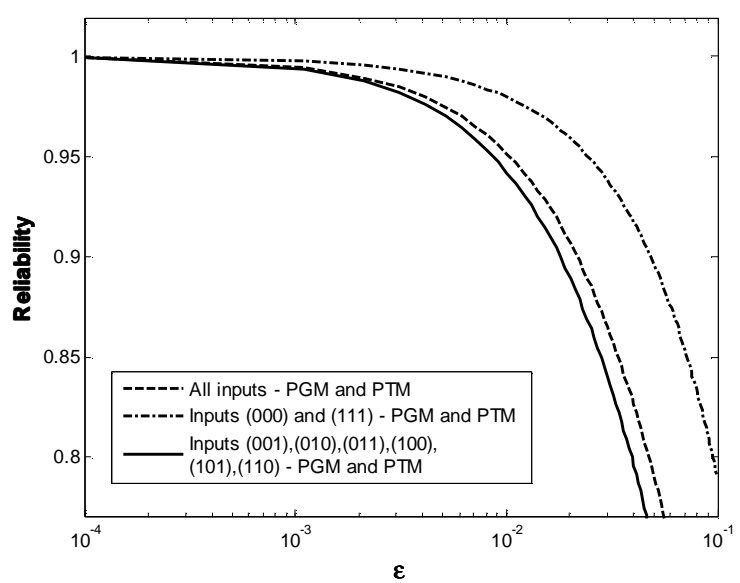

Fig. 4. The reliability of the majority-based full adder.

We also studied a comparator circuit which is shown in Fig. 5. The simulation results are shown in Fig. 6. For the individual outputs, the PGM method provides very good reliability approximations of the exact results obtained by using PTMs. Since the PGM method computes each output reliability independently and uses an identical formulation for all input patterns, it only requires space and time complexities that grow with $n$ and $n \bullet 2^{m}$, respectively. In contrast, for the PTM method, space and runtime complexities grow with at least $2^{m+n}$.

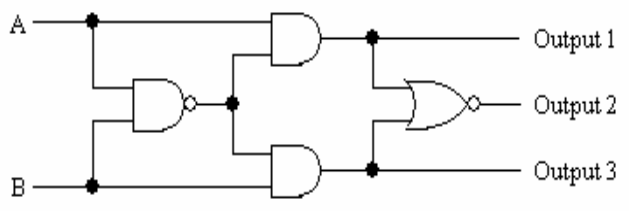

Fig. 5. A comparator circuit.

For the overall circuit reliability, however, the PGM method produces less accurate approximations. This is a 
result of using the product of individual output reliabilities to get an overall reliability. In the case of the comparator circuit, the PGM method would produce its worst approximation if its outputs were generated directly from a fanout. Consider the two output signals of a faultfree fanout. If the input has probability 0.5 of being " 0 " or " 1 ", both of its outputs will be either " 0 " or " 1 " with probability 0.5 and the probability that one output is " 0 " while the other is " 1 " will be zero. Therefore, the probabilities for the output combinations $(00,01,10,11)$ are $(0.5,0,0,0.5)$. In the PGM evaluation method, however, the two output signals are considered to be independent so the probabilities for the output combinations $(00,01,10,11)$ become $(0.25,0.25,0.25$, 0.25 ) by multiplying the individual reliabilities. While independently the output reliabilities would be correct, multiplying them together gives an incorrect overall reliability since the outputs are not independent. The comparator circuit is similar to this worst-case scenario but is a slight improvement since output 2 is generated from a gate output and not directly from a fanout.

For all of the circuits we have considered the discrepancy between the PGM and PTM method never exceeds that of this worst-case scenario where all outputs are generated by a single fanout. Actually, in many cases this discrepancy is very small (see [12]).

\section{CONCLUSION}

The feasibility of emergent nanotechnologies for logic circuit design hinges on our ability to understand the minimum reliability requirements needed for individual gates as well as on our ability to correctly estimate the reliability of circuits with multiple gates. We have described two related approaches to address these challenges. The PGM approach has been used to derive gate error bounds and can also be used to provide approximate reliability results with computational costs that grow exponentially with the number of circuit inputs. The PTM approach proposed by Krishnaswamy et al. is mathematically accurate at higher computational cost, growing exponentially with both the number of inputs and outputs. The errors incurred by PGM are relatively small for the circuits investigated in this paper and in [12]. Whether and when PGM estimates would be sufficiently accurate for purposes of design and engineering of nanoscale circuits is an open question. Future work will investigate whether it is possible to bound or characterize the error incurred by PGM. In addition, further investigations will be made towards computationally efficient reliability estimation methods applicable to very large logic circuits, including circuits with feedback loops.

\section{ACKNOWLEDGEMENT}

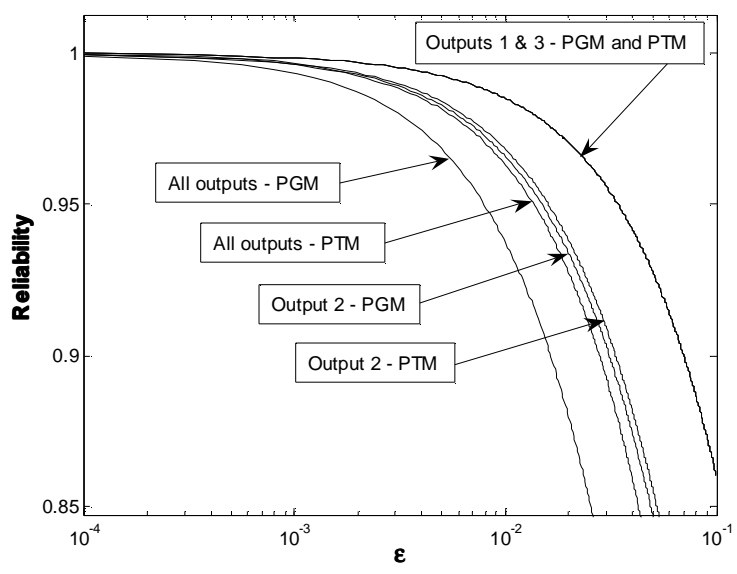

Fig. 6. The reliability of the comparator.

This work is supported by the NASA award no. NCC 2-1363 and National Science Foundation ITR Grant No. 0135946.

\section{REFERENCES}

[1] S.K. Shukla and R.I. Bahar, Nano, Quantum and Molecular Computing: Implications to High Level Design and Validation, Kluwer Academic Publishers, 2004.

[2] J. Han, Fault-Tolerant Architectures for Nanoelectronic and Quantum Devices, Ph.D. dissertation, Delft University of Technology, 2004. http://www.qi.tnw.tudelft.nl/ jie

[3] J. Han and P. Jonker, "A defect- and fault-tolerant architecture for nanocomputers," Nanotechnology, vol. 14, no. 2, pp. 224-230, 2003.

[4] R.I. Bahar, J. Chen and J. Mundy, "A probabilistic-based design for nanoscale computation," Chapter 5 in [1].

[5] J.B. Gao, Y. Qi and J.A.B. Fortes, "Bifurcations and fundamental error bounds for fault-tolerant Computations," IEEE Tr. Nanotechnology, (in press).

[6] S. Krishnaswamy, G.F. Viamontes, I.L. Markov and J.P. Hayes, "Accurate reliability evaluation and enhancement via probabilistic transfer matrices," DATE'05.

[7] T. Oya, T. Asai, T. Fukui and Y. Amemiya, "A majoritylogic device using an irreversible single-electron box," IEEE Tr. Nanotechnology, vol. 2, no. 1, pp. 15-22, 2003.

[8] C.S. Lent and P.D. Tougaw, "A device architecture for computing with quantum dots," Proc. IEEE, vol. 85, pp. 541-557, 1997.

[9] M.B. Tahoori, J. Huang, M. Momenzadeh and F. Lombardi, "Defects and faults in quantum cellular automata at nanoscale," 2004 IEEE VLSI Test Symp. 
[10] M. Sulieman and V. Beiu, "On single electron technology full adders," IEEE Nano 2004, pp.317-320, 2004.

[11] R. Zhang, K. Walus, W. Wang and G.A. Jullien, "A method of majority logic reduction for quantum cellular automata," IEEE Tr. Nanotechnology, vol. 3, no. 4, pp. 443-450, 2004.

[12] J. Han, E. Taylor, J. Gao and J. Fortes, "Faults, error bounds and reliability of nanoelectronic circuits," ASAP05. 